\title{
O Uso de Visualizadores Portáteis como Fator de Aumento na Produtividade da Construção Civil
}

\author{
The Portable Viewers Use as Increase Factor in Productivity of Civil Construction \\ Max Andrade \\ UFPE, Brazil \\ Mikael Brochardt \\ UFPE, Brazil \\ max.andrade@ufpe.br \\ kall_muller@outlook.com \\ - Jonas Assis \\ UFPE, Brazil \\ jonas.henrique@ufpe.br
}

\begin{abstract}
The potential of Building Information Modeling (BIM) as booster of productivity in construction can be better explorer using solutions of information visualization in digital environment, using tablets and smartphones. The maintenance of the project in digital environment at the construction site has several advantages over the use of printed designs, enabling improvements in the flow, quality and quantity of information in the enforcement phase. In BIM, this solution is further efficient, exploiting its wealth of information. This article examines portable visualization tools concerning resources and suitability for use in construction sites, forming a comparative and suggestive overview of these applications.
\end{abstract}

Keywords: BIM, Visualization, Information, Application, Productivity

\section{Introdução}

O Building Information Modeling (BIM) ao potencializar o fluxo das informações do edifício, pode auxiliar no incremento da produtividade na construção civil. As ferramentas aprimoradas de visualização e compatibilização de sistemas prediais atreladas ao BIM podem contribuir com soluções que levem a redução de erros de projeto que resultariam em posteriores erros construtivos e retrabalhos. Apesar desse potencial, o BIM tem sido implementado no Brasil de forma lenta e incompleta, resultando, em alguns casos, em ganhos de produtividade abaixo do verdadeiro potencial do mesmo.

Uma breve análise comparativa da realidade brasileira revela que alguns fatores têm travado a implementação do BIM, como a falta de capacitação dos projetistas, a dificuldade em mudar os processos tradicionais de projeto e o modo como a informação chega ao canteiro de obras. Tradicionalmente, a informação chega ao canteiro de obras por meio de plantas impressas. Essas além de conterem informações abstratas, muitas vezes, difíceis de entender, ainda são incompletas, factíveis de erros e difíceis de serem alteradas. Mesmo ao se trabalhar com modelos BIM complexos e ricos de informação, ao transformar a documentação em plantas, cortes e fachadas, muitas das informações "penduradas" no modelo BIM se "perdem".

No modelo BIM é possível armazenar as informações sobre os componentes da construção (Goes e Santos, 2011). $\mathrm{O}$ processo de modelagem em softwares de autoria BIM, por ser mais complexo e preciso, acaba por demandar mais tempo em relação ao trabalho em CAD (Computer Aided Drafting). Apesar disso, o modelo gerado contém uma riqueza de informações que outrora eram distribuídos em vários desenhos bidimensionais de projetos hidráulicos, estruturais, elétricos, etc. A unificação dos projetos em um modelo único permite a identificação e solução de conflitos durante o próprio processo de modelagem, além de melhorar a qualidade da informação do edifício.

Apesar de apresentar maior coerência e qualidade da informação em relação aos métodos de CAD tradicionais, os desenhos gerados a partir do modelo BIM para uso na obra ainda são abstrações simbólicas bidimensionais, que desperdiçam parte da riqueza de informações do edifício.

Uma forma relativamente barata de melhorar o fluxo da informação no canteiro de obras é através do uso de dispositivos móveis (smartphones e tablets) para a visualização das informações dos diferentes projetos. Por meio desses dispositivos é possível visualizar em tempo real informações atualizadas e precisas sobre a obra, com toda a riqueza semântica que pode estar contida no modelo BIM. Também é possível enviar informações para os escritórios de projeto sobre eventuais alterações necessárias no projeto e receber em tempo real as alterações sofridas no modelo BIM. Dessa forma, melhora-se o fluxo da informação no canteiro de obras, em relação ao uso de plantas impressas, por fornecer um modelo completo e menos 'abstrato' do edifício. 
Com o uso de novas ferramentas de visualização digital do edifício facilita-se a compreensão do projeto no canteiro de obras. As ferramentas de visualização que utilizam modelos BIM, além de permitirem a extração de informações geradas e a gestão de projeto, permitem também, que outros envolvidos possam acompanhar o seu desenvolvimento de várias formas. Assim, é possível aumentar o número de envolvidos com a visualização, principalmente em dispositivos móveis, dada a facilidade de acesso, manuseio intuitivo e o desempenho dos softwares para essa plataforma.

Os aplicativos de visualização, por incorporarem o Modelo BIM, agregam em um único modelo informações relacionadas aos vários sistemas do edifício. De posse dessas informações, o aplicativo é capaz de permitir a visualização isolada de cada sistema, bem como, a sua contextualização compatibilizada com os demais sistemas. Assim, é possível filtrar a informação de acordo com as áreas de interesse de cada envolvido no projeto. Essa característica permite, por exemplo, que o responsável técnico de hidráulica analise o projeto de tubulações isoladamente, mas tendo a opção de ver sua relação e possíveis conflitos com o projeto de alvenaria. Esse mesmo modelo permite gerar outros filtros, bem como, a geração imediata de novos cortes bidimensionais e vistas tridimensionais.

Já os aplicativos de realidade aumentada oferecem um novo modo de visualizar o edifício, pois utilizam simulações menos abstratas que aquelas usadas nas plantas impressas. É possível criar a ilusão da construção real, contextualizada com o espaço físico do canteiro de obra, por meio de um modelo tridimensional projetado no dispositivo móvel.

No presente trabalho foram avaliados alguns dos principais aplicativos de visualizaçãoe de realidadeaumentada para smartphones e tablets existentes no mercado. Esses foram testados e classificados de acordo com as características da tecnologia utilizada, as plataformas suportadas, os tipos de licença, a qualidade da informação apresentada, o fluxo da informação, a capacidade de atualização do modelo, a possibilidade de sincronização da informação, a facilidade de uso da informação no canteiro de obra, o custo de implementação, entre outros fatores.

Esses testes iniciais permitiram que fossem identificados os aplicativos que possuem características que os tornam atualmente adequados ao uso no canteiro de obras. Dessa forma, os aplicativos que se adequam ao uso na obra serão testados também quanto ao seu desempenho.

O produto final do artigo é uma análise comparativa e sugestiva dos principais aplicativos para smartphones e tablets para visualização de informação de projeto nos canteiros de obra, servindo, portanto, de base para decisões relacionadas à implementação BIM e revisão da forma de disposição da informação de projeto no canteiro de obras.

É dentro desse contexto que esse artigo se insere. O objetivo, portanto, é mostrar um estudo comparativo, que vem sendo feito numa pesquisa de Iniciação Científica, sobre os principais softwares de visualização do modelo do edifício para smartphones e tablets factíveis de serem usados nos canteiros de obras.

\section{Procedimentos Metodológicos}

O ponto de partida foi a identificação dos principais aplicativos de visualização do modelo do edifício para smartphones e tablets disponíveis no mercado para plataforma iOS (Apple) e Android (Google). Para esse fim, foi feito um levantamento das ferramentas existentes nas seguintes categorias: aplicativos de visualização 2D/3D, aplicativos BIM, aplicativos de gerenciamento de projeto e aplicativos de realidade aumentada. Desse modo, foram identificados os seguintes aplicativos: AR-Player, AutoCAD 360, Graphisoft BIMx, ProjectWise Explorer Mobile, Shimmer, Sketchup Mobile Viewer, Tekla Field 3D e Trimble ProjectSight.

Dentre os aplicativos de visualização identificados, também foram avaliados quais deles incorporavam simplesmente a geometria do modelo e quais representavam o Protótipo Virtual do edifício por meio de um Modelo BIM. Para esses últimos procurou-se identificar se as informações geradas no modelo BIM podem ser efetivamente extraídas no canteiro de obras, por meio de smartphones e/ou tablets. Além do mais, buscouse avaliar se as informações oriundas desses dispositivos são realmente úteis para serem utilizadas no canteiro de obras ou se a simples utilização de um modelo geométrico pode fornecer as informações necessárias e suficientes de projeto. Essa avaliação permitiu perceber quão positiva pode ser a mudança no modo de visualização de projeto sugerida nesse trabalho. Também buscouse identificar como se dá o fluxo da informação nos aplicativos em estudo, (unidirecional ou bidirecional), e se estes oferecem atualizações do modelo em tempo real. Por fim, buscou-se avaliar se esses aplicativos (sejam de visualização e/ou de realidade aumentada) podem substituir (parcial ou completamente) o papel impresso nos canteiros de obras da construção civil brasileira. A forma empregada para fazer esses tipos de avaliação foi por meio de testes dos aplicativos e na literatura vinculada aos mesmos. Para esse momento da pesquisa ainda não foram feitos experimentos no canteiro de obras.

Após terem sido definidos os softwares a serem usados na pesquisa, foram selecionados aqueles que possuem maior potencial de uso no canteiro de obras. A escolha desses aplicativos se deu por meio da avaliação daqueles que pudessem extrair a maior quantidade de informações necessárias e úteis para serem usados no canteiro de obras. Escolhidos os aplicativos esses foram submetidos a testes de usabilidade e desempenho, empregando os seguintes projetos, equipamentos e procedimentos:

- Equipamentos utilizados:

1. Tablet Samsung Galaxy Tab 3 Lite (Android, 1GB de RAM, Processador Dual-Core de $1.2 \mathrm{GHz}$ )

2. Tablet CCE Motion Tab (Android, 1 GB de RAM, Processador One-Core de $1.2 \mathrm{GHz}$ )

- Modelos utilizados no teste: (Para que o projeto tivesse uma maior abrangência foram criados 3 modelos de edifício) 
1. Edifício de pequeno porte - cerca de $50 \mathrm{~m} 2$

2. Edifício de médio porte - cerca de $500 \mathrm{~m} 2$

3. Edifício de grande porte - cerca de $9.000 \mathrm{~m} 2$

- Procedimentos utilizados no teste:

1. Modelagem/desenho do edifício nos respectivos softwares

2. Abertura do modelo/planta do edifício

3. Movimentação e extração das informações que podem ser extraídas do modelo

4. Verificação da facilidade de manuseio do modelo a partir do desempenho do mesmo

5. Análise comparativa dos softwares analisados

Embora seja fundamental o estudo prático do uso desses aplicativos nos canteiros de obras, o trabalho a ser apresentado neste artigo se limitará a uma análise comparativa da viabilidade do uso desses aplicativos no canteiro de obras, a partir do conhecimento das características de funcionamento dos mesmos. Não serão, portanto, apresentados os resultados dos estudos da implantação desses aplicativos no canteiro de obras, haja vista, essa pesquisa ainda estar em estágio inicial. Os testes de implementação estão planejados para as fases seguintes da pesquisa.

Fluxo da Informação Através das Pranchas e por Meio Digital

O fluxo de informação e as pranchas no canteiro de obras A inserção de novas tecnologias no processo de projeto/ construção pode tornar-se um importante fator de aumento de produtividade da construção civil, especificamente ao contribuir com a melhoria do fluxo da informação. Para Nascimento e Santos (2003) a informação é uma das matériasprimas mais importantes na cadeia produtiva, sendo tão importante quanto os insumos utilizados na produção. Os mesmos autores afirmam que a falta de clareza, bem como a insuficiência de informação, gera no canteiro de obras erros e imprecisões executivas que levam a atrasos e retrabalhos, afetando a produtividade e a qualidade do produto final.

O modelo atual de distribuição da informação no canteiro deobra, por meio de plantas impressas tem se mostradoineficaz quando comparado a modelos usados em outras indústrias. $O$ desenvolvimento de projetos em CAD traz poucas melhorias em termos de fluxo da informação comparados aos projetos outrora desenvolvidos à mão nas antigas pranchetas dos escritórios de arquitetura e engenharia civil. Isso se dá devido à manutenção da lógica de desenho representativo e do modo de saída de informação para o canteiro, a saber, em plantas impressas. Entre as desvantagens das plantas impressas, podese destacar:

- Ocorrência de erros de representação e incoerências entre os desenhos, devido à confecção das partes do edifício de maneira independente em CAD;

- $\quad$ Alto custo de impressão a cada revisão;

- Feedback lento e ineficaz, pois o uso de plantas impressas dificulta o retorno de anotações solicitando esclarecimentos e revisões;

- Dificuldades na organização do grande número de plantas;

- Inadequação das grandes plantas impressas à movimentação dentro do canteiro.

\section{O exemplo de outros setores}

Vários setores da economia têm obtido bons resultados na utilização de dispositivos móveis na organização do fluxo de informações. Tablets já são usados com bons resultados nas áreas de comércio, educação, empresarial, entre outros (FONTES; SOARES, 2012). O uso desses dispositivos tem trazido ganhos significativos de produtividade com baixo custo. Tais dispositivos possuem diversos recursos que podem ser usados no aumento da produtividade, como, por exemplo, acesso à Internet. Esse recurso permite a sincronização de dados, evitando retrabalhos no registro de informações (FONTES; SOARES, 2012). Segundo Neto (2013), citando pesquisa realizada em 2012, quatro em cada cinco empresas de TI (Tecnologia da Informação) norte-americanas já trabalham com dispositivos móveis. 0 mesmo autor ainda demonstra que esses dispositivos já têm sido usados com sucesso nas áreas de saúde, transportes, educação e administração (NETO; RUSCHEL; PICCHI, 2013). Fica claro que o uso de dispositivos móveis permite um aumento significativo da quantidade e qualidade da informação, propiciando ganhos de produtividade em diversos setores econômicos.

\section{Softwares de Visualização Usados em Canteiro de Obras}

\section{A escolha dos softwares}

Em um primeiro momento da pesquisa, foram identificados todos os softwares que ofereciam algum tipo de visualização dos arquivos utilizados hoje nos projetos de construção civil. A investigação foi realizada nas lojas de aplicativos das 3 principais plataformas em uso no Brasil: Android (Google), iOS (Apple) e Windows Phone (Microsoft). Foram usadas palavras-chaves como "BIM", "Realidade Aumentada" e "CAD", além de navegar pelos softwares relacionados em cada um dos aplicativos encontrados. Também foram realizadas buscas em ferramentas de pesquisa online, como o Google, e visitas aos sites das principais empresas de software para construção civil, como a Autodesk, Graphisoft, Tekla, Trimble e Bentley, buscando descobrir se essas também produziam aplicativos móveis. Dentro desse conjunto maior foi realizada uma investigação preliminar de quais desses softwares tinham potencial para serem usados no canteiro de obras, por apresentarem informações adequadas de modo adequado para a fase executiva do projeto. Foram dispensados softwares com bons recursos que se aplicam prioritariamente às fases de concepção e discussão do projeto, como, por exemplo, os softwares 
de desenho CAD e Realidade Virtual. Dessa forma, foram selecionados softwares de visualização 2D, 3D, de Realidade Aumentada (R.A.) e softwares que utilizam modelos desenvolvidos em softwares de autoria BIM.

Os visualizadores de representação $2 \mathrm{D}, 3 \mathrm{D}$ e em R.A. possuem recursos com grande potencial de uso no canteiro, motivos dos quais também foram selecionados. Alguns também possuem uma maior maturidade de desenvolvimento que os aplicativos BIM, por estarem há mais tempo no mercado. Dessa forma, uma análise comparativa pode revelar recursos capazes de agregar qualidade aos visualizadores BIM.

\section{Descrição dos softwares}

Dentro do grupo de aplicativos de visualização 2D/3D foram identificados os seguintes softwares: AutoCAD 360 e Sketchup Mobile Viewer. No conjunto de aplicativos de Realidade Aumentada, há de se destacar os seguintes: ARPlayer e Shimmer. Os principais aplicativos de gerenciamento

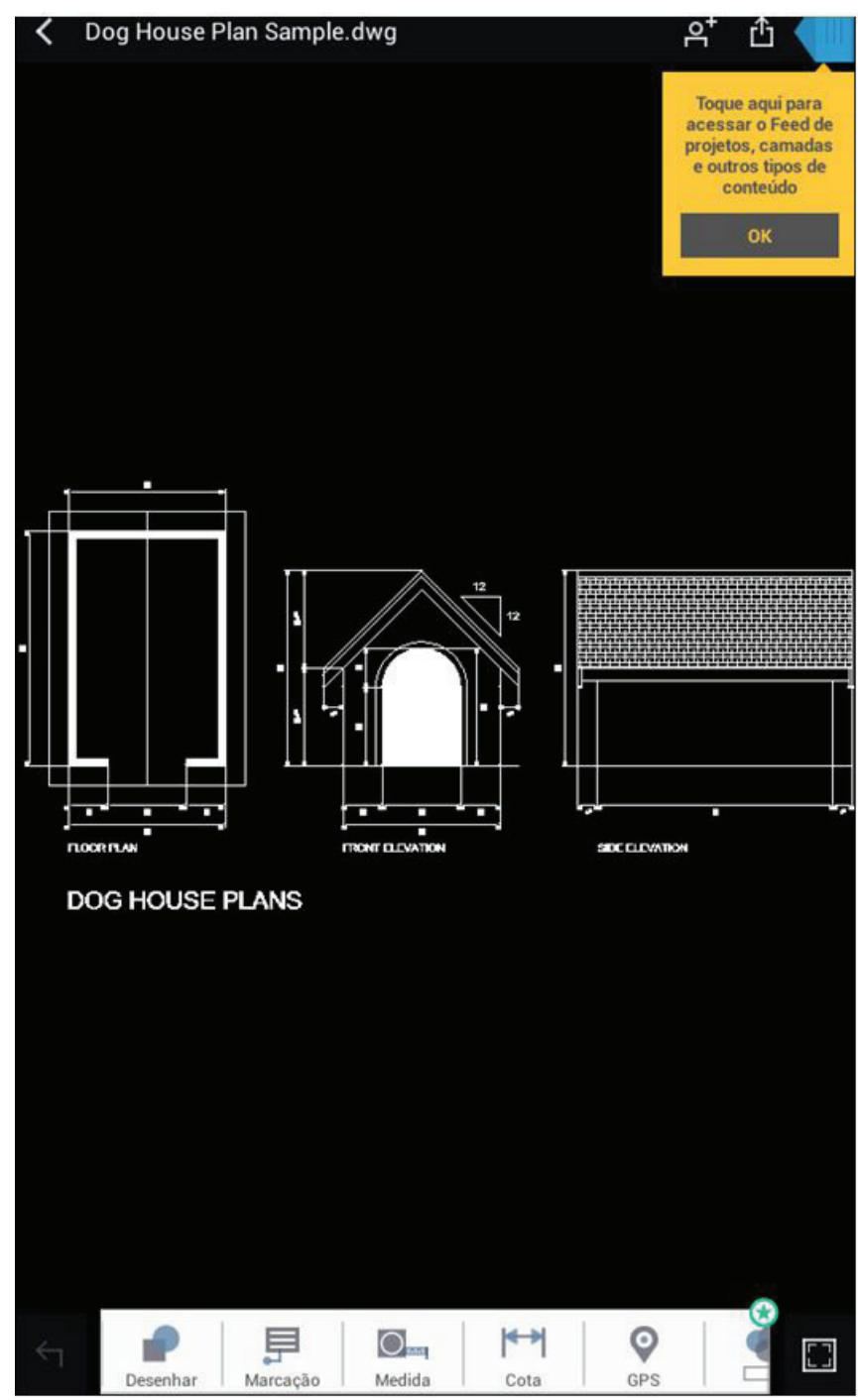

Imagem 1: Ambiente de visualização do AutoCAD 360, com destaque para as instruções mostradas nas primeiras utilizações. de projeto foram: Trimble ProjectSight e ProjectWise Explorer Mobile. E os principais softwares BIM identificados foram: Graphisoft BIMx e Tekla Field 3D.

AutoCAD 360 (Autodesk): Trata-se de um software desenvolvido pela Autodesk para visualização de arquivos $\mathrm{CAD}$ em dispositivos móveis. Faz uso de desenhos 2D e 3D de representação, mas não incorpora informações ao modelo (BIM). Mesmo com a possibilidade de exibição de arquivos CAD 3D, seu foco de atuação são os desenhos 2D desenvolvidos no AutoCAD (Arquivos DWG). Permite utilizar o sistema GPS do dispositivo para localizar o usuário nos ambientes do projeto, caso este esteja devidamente geolocalizado. Está disponível nas plataformas Android e iOS, permitindo a utilização em tablets e smartphones. Pode ser utilizado gratuitamente, mas oferece versão paga ( $\mathrm{R} \$ 127,33$ por ano) com recursos adicionais, como abertura de arquivos maiores que $10 \mathrm{mb}$ e criação de novos desenhos. As informações apresentadas dependem dos desenhos disponíveis no Projeto Executivo, podendo incluir plantas, cortes, fachadas, elevações, detalhes, etc. A visualização é facilitada pelas opções de medição e gerenciamento de layers. Permite o fluxo bidirecional de informações por meio do envio de notas de revisão e atualização sincronizada do projeto por meio da internet.

Sketchup Mobile Viewer (Trimble): Software criado pela Trimble visando possibilitar a visualização de modelos SKP (Trimble Sketchup) em dispositivos móveis. Permite também a exibição 3D dos modelos disponíveis no banco de dados da empresa, o 3D Warehouse. Tem suporte às plataformas Android e iOS, usadas em tablets e smartphones. O software é pago, custando R\$29,79. Apresenta um modelo 3D geométrico, que não agrega informações, como ocorre no Sistema BIM. O fluxo de informações é unidirecional, pois não oferece a possibilidade de atualização e adição de notificações para revisão.

AR-Player (Inglobe): Software proprietário da Inglobe Technologies destinado à visualização em Realidade Aumentada de modelos geométricos 3D. Funciona em Tablets e Smartphones com sistemas Android e iOS. Seu uso é totalmente gratuito (Freeware). Abre arquivos próprios do software (.armedia) que podem ser gerados a partir de plugins do 3DS Max, Sketchup, Scia Engineer, Vectorworks, Maya e Cinema 4D. O aplicativo não permite a obtenção de informações adicionais do modelo, como ocorre no BIM, nem uma interação completa com o modelo, pois não permite geração de cortes nem gerenciamento de layers. Porém, oferece uma experiência diferenciada dos demais softwares, por usar um recurso gráfico diferenciado, a Realidade Aumentada. Não permite fluxo de informações através da rede, atualizando arquivos do projeto, e a forma de apresentação de modelo não favorece seu uso na obra, pois não há possibilidade de gerar desenhos bidimensionais como cortes e plantas. 


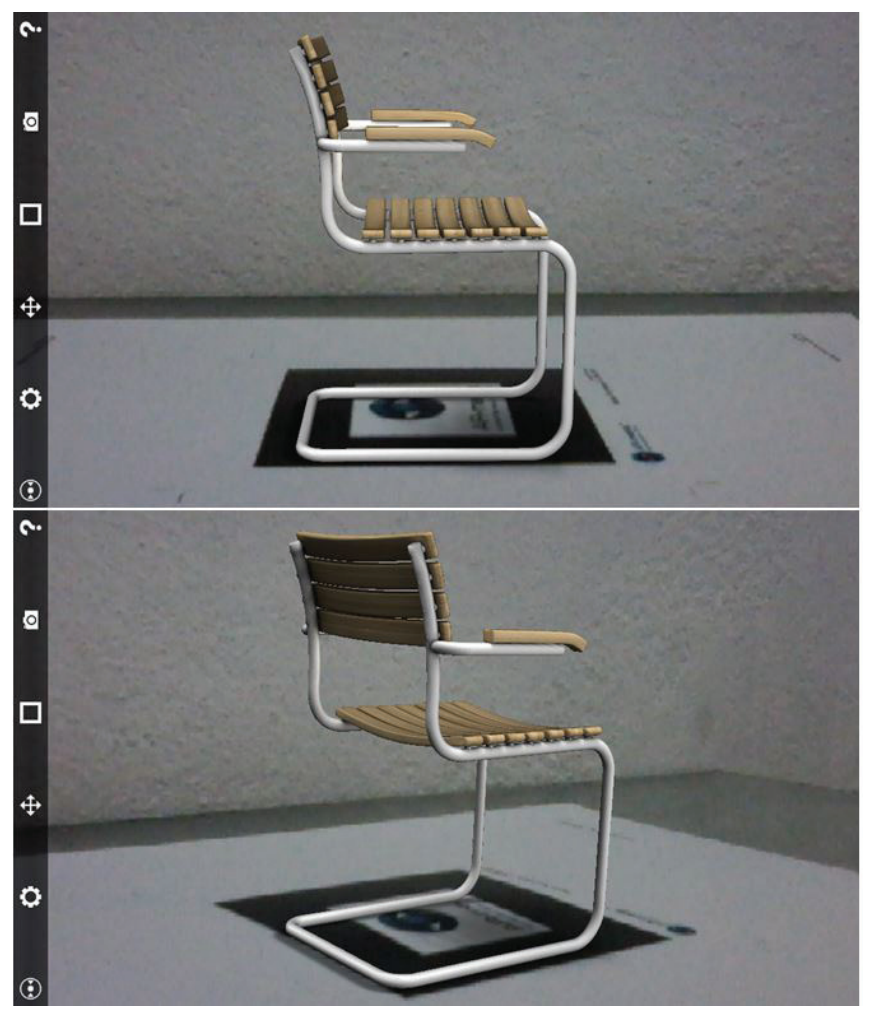

Imagens 2 e 3: R.A. sobre marcador com AR-Player, com destaque para a possibilidade alterar a visualização movendo o dispositivo.

Shimmer (ionReality): Aplicativo de Realidade Aumentada para tablets e smartphones com iOS comercializado ao custo de $\mathrm{R} \$ 3,00$. Trabalha com os formatos DAE (Collada), OBJ (Wavefront),3DS(3DStudio)e X(DirectX). Nãopermiteo acesso a informações do modelo virtual do edifício. Trabalha com fluxo unidirecional de informações por meio da sincronização com serviço de armazenamento em nuvem (Dropbox). Permite utilizar cédulas monetárias como marcadores para a projeção da Realidade Aumentada, facilitando seu uso no dia-a-dia.

BIMx (Graphisoft): O BIMx é o aplicativo de visualização de modelos BIM em dispositivos móveis desenvolvido pela Graphisoft. Oferece a visualização tridimensional do modelo BIM, permitindo a extração de informações sobre a materialidade dos componentes do edifício. Funciona em sistemas Android e iOS sob licença Shareware, sendo que a versão completa ( $\mathrm{R} \$ 117,48)$ adiciona recursos como a possibilidade de visualizar os documentos 2D do modelo. Utiliza arquivos específicos (.bimx) exportados do Graphisoft Archicad. Permite a geração imediata de cortes no modelo 3D e a extração de informações como altura, espessura e material das vedações, descrição dos objetos (janelas, portas) com informações úteis como altura do peitoril, dimensões do vão, etc. Oferece possibilidades de fluxo bidirecional da informação, além de opções avançadas de visualização, como 3D Anaglifo e navegação em primeira pessoa pelos ambientes do modelo.

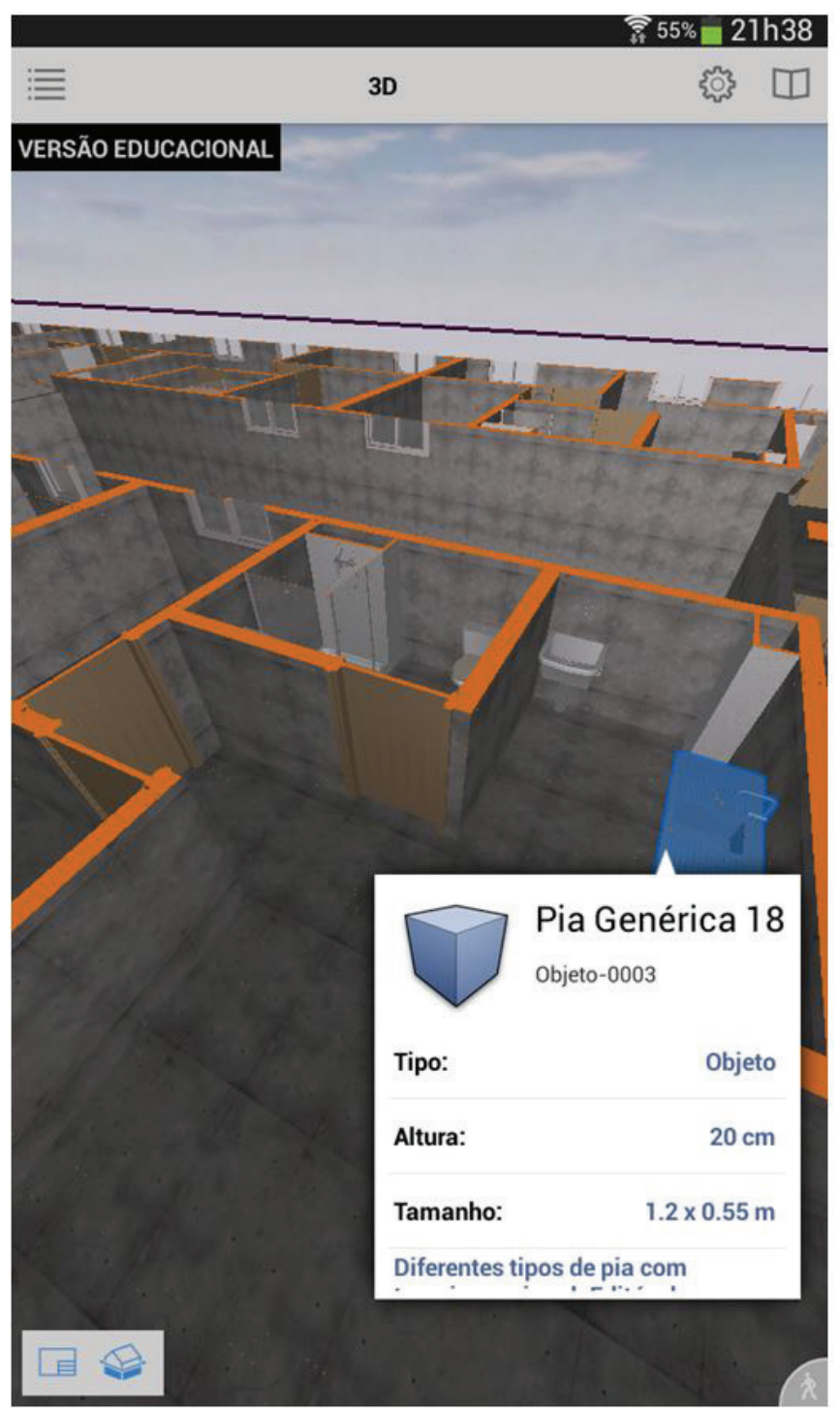

Imagem 4: Visualização de corte tridimensional no BIMx com destaque para consulta de informações sobre os elementos.

Field 3D (Tekla, Trimble): Aplicativo da Tekla destinado à visualização tridimensional de projetos BIM, sendo disponibilizado para smartphones e tablets que utilizem sistema Android ou iOS sob licença Shareware. A versão completa custa $R \$ 124,8$ por trimestre e ativa o fluxo de informações bidirecional do software, que passa a permitir a sincronização em nuvem e a adição de notas no projeto, além de oferecer suporte a mais formatos de arquivos, como o DWG, não suportado pela versão gratuita, focada em arquivos IFC. Permite a visualização tridimensional isolada dos sistemas prediais (instalações hidráulicas, elétricas, estrutura). Permite também a ocultação de layers ou objetos específicos, além da obtenção de informações diversas como altura e altimetria das vedações e material constituinte. 


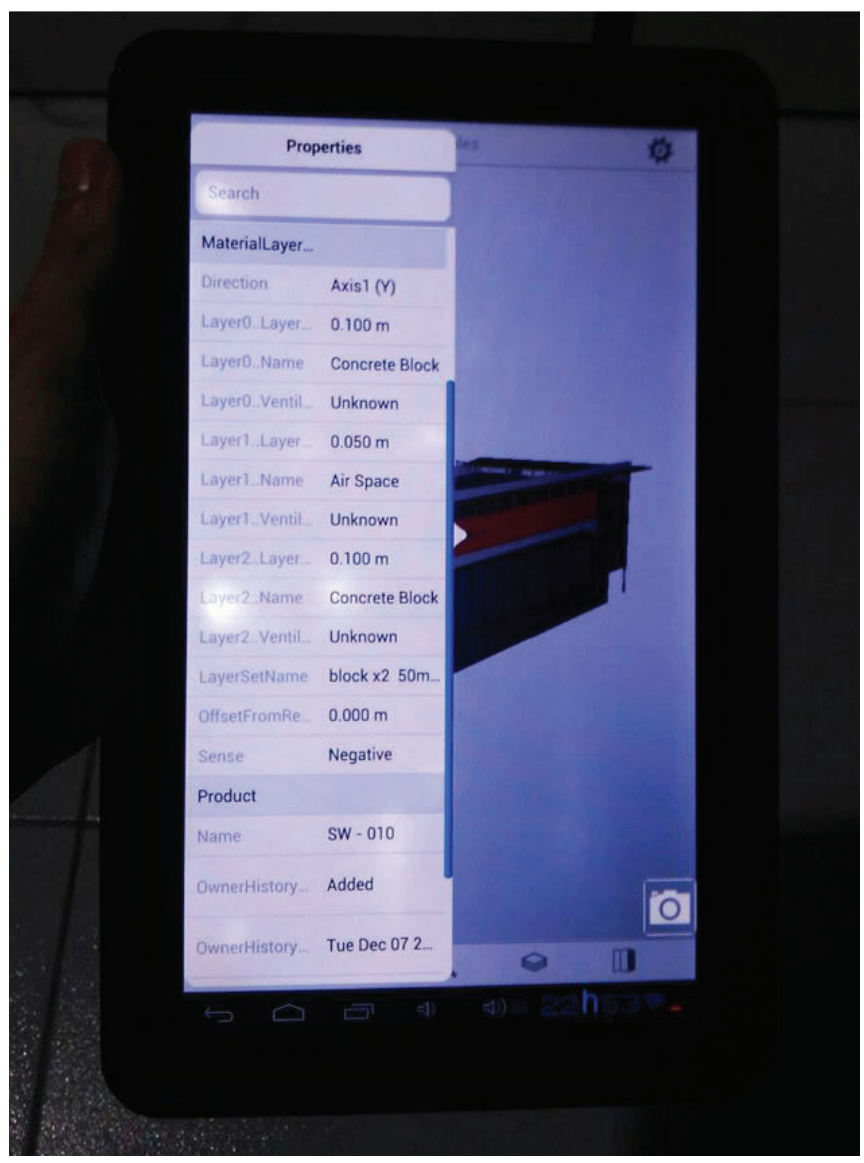

Imagem 5: Ambiente de visualização 3D do Field 3D com consulta de informações sobre os elementos, com destaque para a grande quantidade de informações disponíveis, ainda que sem um tratamento adequado de apresentação ao usuário.

ProjectWise Explorer Mobile (Bentley): Software móvel criado pela Bentley para trabalhar em conjunto com o ProjectWise, sua plataforma de gerenciamento de projeto que inclui diversos programas de publicação, revisão e acompanhamento de projeto. Disponível apenas para iOS, é distribuído gratuitamente e permite a prévisualização dos diversos arquivos utilizados na fase executiva do projeto, desde imagens, plantas em PDF e arquivos BIM. Seu objetivo consiste em ser um módulo de campo do ProjectWise, sendo assim, trabalha com fluxo de informações bidirecional.

ProjectSight (Trimble): Aplicativo online de gerenciamento de projeto da Trimble. Funciona apenas em iOS e tem por objetivo encurtar a distância entre o canteiro e o escritório. Trabalha sob licença paga no valor de $\mathrm{R} \$ 62,40$. Cria um ambiente colaborativo de compartilhamento de informações, permitindo inclusive o retorno de informações textuais ou fotográficas para o escritório, em um fluxo bidirecional da informação.

\section{Classificação dos Softwares}

\begin{tabular}{|l|l|l|l|}
\hline Softwares & Foco & Plataforma & Licença \\
\hline $\begin{array}{l}\text { AutoCAD } \\
360\end{array}$ & 2D/3D & iOS/Android & Shareware \\
\hline $\begin{array}{l}\text { Sketchup } \\
\text { Mobile }\end{array}$ & 3D & iOS/Android & Paga \\
\hline AR-Player & R.A. & iOS/Android & Freeware \\
\hline Shimmer & R.A. & iOS & Paga \\
\hline $\begin{array}{l}\text { Trimble } \\
\text { ProjectSight }\end{array}$ & Gestão & iOS & Paga \\
\hline $\begin{array}{l}\text { ProjectWise } \\
\text { Explorer } \\
\text { Mobile }\end{array}$ & Gestão & iOS & Freeware \\
\hline $\begin{array}{l}\text { Graphisoft } \\
\text { BIMx }\end{array}$ & 2D/3D/BIM & iOS/Android & Shareware \\
\hline $\begin{array}{l}\text { Tekla Field } \\
\text { 3D }\end{array}$ & 3D/BIM & iOS/Android & Shareware \\
\hline
\end{tabular}

Tabela 1: Classificação dos softwares.

\section{Análise de desempenho dos softwares}

Dos softwares usados na etapa de catalogação da pesquisa, foram selecionados aqueles mais adequados ao uso no canteiro de obras. No próximo item são apresentados os critérios usados para a escolha. Considerando que esses aplicativos têm potencial de uso na obra, os testes empreendidos avaliaram o desempenho e a funcionalidade dos mesmos.

Nessa fasede testes foramusados os seguintes procedimentos de avaliação:

- Equipamentos utilizados:

1. Tablet Samsung Galaxy Tab 3 Lite (Android, 1GB de RAM, Processador Dual-Core de $1.2 \mathrm{GHz}$ )

2. TabletCCEMotion Tab(Android,1GBde RAM, Processador One-Core de $1.2 \mathrm{GHz}$ )

- Modelos utilizados no teste: (Para que o projeto tivesse uma maior abrangência foram criados 3 modelos de edifício)

1. Edifício de pequeno porte - cerca de $50 \mathrm{~m} 2$

2. Edifício de médio porte - cerca de $500 \mathrm{~m} 2$

3. Edifício de grande porte - cerca de $9.000 \mathrm{~m} 2$

- Procedimentos utilizados no teste:

1. Modelagem/desenho do edifício nos respectivos softwares

2. Abertura do modelo/planta do edifício

3. Movimentação e extração das informações que podem ser extraídas do modelo

4. Verificação da facilidade de manuseio do modelo a partir do desempenho do mesmo

5. Análise comparativa dos softwares analisados

Escolha dos principais softwares de visualização

Dentre os softwares analisados, identificou-se que apenas 3 
deles poderiam ter um uso mais difundido no canteiro de obras: AutoCAD 360, BIMx e Field 3D. Esses aplicativos possuem características e recursos que permitem e facilitam sua inserção na obra em substituição das plantas impressas. Foi notado que o Sketchup Mobile Viewer é um software mais genérico, sem tratamento específico para o canteiro de obras. Mas seu potencial para uso no canteiro de obras consiste na sua compatibilidade com as maquetes digitais amplamente usadas hoje na fase de anteprojeto, tornando possível aproveitar esse modelo já existente no canteiro. O AR-Player também se mostrou uma solução de visualização genérica, mas sua tecnologia possui grande potencial de uso no canteiro de obras e já pode ser usada em casos específicos.

Os aplicativos apresentaram os seguintes resultados:

AutoCAD 360: O software demonstrou desempenho satisfatório em todos os dispositivos com plantas de diversos tamanhos. Por oferecer visualização bidimensional, exige menos do dispositivo. $\mathrm{O}$ aprendizado do software é rápido e suas funcionalidades são categorizadas e organizadas de modo lógico, oferecendo acesso rápido às principais funções, como a extração de medidas. Além disso, a primeira utilização é acompanhada por mensagens que guiam a exploração das principais opções do aplicativo. Porém, a utilização do servidor do software não é vinculada ao aplicativo, sendo necessário o acesso por meio de um computador ou por meio de um software complementar. Apesar das vantagens de obter projetos do servidor online, a importação do armazenamento do dispositivo não é possível, fazendo com que a velocidade de importação dependa da qualidade da conexão com a internet. Sua versão gratuita não permite a abertura de arquivos maiores que $10 \mathrm{mb}$, e na versão paga esse limite é estendido aos 30mb. Apesar da limitação, a maioria das plantas usadas na construção não ultrapassam esses valores.

BIMx: O BIMx foi capaz de importar imediatamente os modelos a partir do armazenamento do dispositivo. O software mostrou-se bastante fluido na exploração do modelo 3D dos modelos de 50 e $500 \mathrm{~m}^{2}$. A navegação 3D no modelo de $900 \mathrm{~m}^{2}$ apresentou travamentos constantes. Mesmo nos modelos que demostraram fluidez no início da navegação, a experiência não foi plena, pois a geração de cortes 3D apresentou travamentos. Esses travamentos foram esporádicos no modelo de $50 \mathrm{~m}^{2} \mathrm{e}$ constantes no modelo de $500 \mathrm{~m}^{2}$, em ambos os dispositivos. A navegação na documentação $2 \mathrm{D}$ só é possível na versão paga. $\mathrm{O}$ aprendizado do programa é rápido, por apresentar uma interface comum e intuitiva.

Field 3D: O software da Tekla apresentou diversos problemas nos testes de desempenho. No dispositivo Samsung, apesar de possuir maior poder de processamento que o CCE, o aplicativo não funcionou, nem sequer inicializando completamente, revelando uma provável incompatibilidade com alguns dispositivos. No Tablet CCE, o modelo de $50 \mathrm{~m}^{2}$ levou 4 minutos para ser importado e apresentou fluidez satisfatória. As opções de visualização tridimensional são diversas, como a ocultação de elementos individuais, layers e sistemas prediais, além da obtenção de informações diversas sobre esses elementos. Os modelos de 500 e $9.000 \mathrm{~m}^{2}$ não foram carregados, causando fechamento do aplicativo após algum tempo de carregamento. Dessa forma, foi usado um Tablet adicional (Apple iPad) a fim de verificar a fonte do problema. Apesar da capacidade de processamento maior no iPad, os modelos médio e grande não foram carregados, revelando que o aplicativo possui limitação no tamanho dos arquivos, apesar de esse limite não ser informado pelo fabricante.

\section{Comparativo de desempenho e funcionalidade dos softwares adequados para o canteiro de obras}

A análise revelou que há no mercado essencialmente dois aplicativos realmente preparados para o uso no canteiro de obras: AutoCAD360 eBIMx.Éevidentequeoutros aplicativos podem ser explorados em conjunto oferecendo soluções compostas. Porém, esses dois softwares podem ser destacados por serem satisfatórios em diversos aspectos, permitindo o uso de um único software completo. $O$ Field 3D possui diversas características adequadas ao uso em obra, mas as questões de desempenho dificultam seu uso. As opções de visualização em realidade aumentada de softwares como o AR-Player possuem potencial de inserção no canteiro para visualização dinâmica de detalhes construtivos diversos, podendo servir como software adicional. Assim, seu potencial seria explorado apenas como opção adicional de visualização.

Quanto à decisão entre AutoCAD 360 e BIMx é importante destacar que o AutoCAD 360, apesar das qualidades como software, proporciona melhorias na produtividade apenas por meio da mudança do modo como a informação chega ao canteiro. Já o BIMx, carrega consigo um novo sistema de projeto, que agrega informação a um modelo tridimensional, alterando além do modo de inserção da informação, o tipo e a qualidade dessa informação, apesar de necessitar um processo mais complexo de implementação.

\section{Substituição do papel pelo modelo digital como fator de aumento} da produtividade do canteiro de obras

Fica claro que o uso de equipamentos portáteis na construção civil pode trazer ganhos significativos, representando um grande potencial ainda pouco explorado. Esse modelo de fluxo de informações apresenta as seguintes vantagens em relação às plantas impressas:

- Possibilidade de aumento da quantidade de informação sem aumento significativo de custo em comparação às plantas impressas;

- $\quad$ Maior clareza (qualidade) da informação apresentada devido às possibilidades de aproximação, medição do desenho, etc.;

- Seletividadeda informação(possibilidadede isolar elementos e sistemas do desenho) propiciando maior clareza;

- Maior flexibilidade de uso dentro do canteiro de obras;

- Maior durabilidade (segurança contra perdas) e disponibilidade da informação armazenada em meio digital e em nuvem;

- Maior exploração da riqueza de informações do modelo BIM, por meio da visualização tridimensional e geração rápida de novas vistas;

- Integração facilitada entre várias fontes de informação, usando a rede como meio de comunicação e sincronização; 
- Revisões não geram custos adicionais relativos à impressão.

As vantagens da adoção desse sistema sobrepõem desvantagens relacionadas a questões financeiras e de treinamento, pois os resultados compensam os investimentos, já em curto prazo. Alguns exemplos, como o da Construtora Frechal (MOBUSS CONSTRUÇÃO, 2014), comprovam a viabilidade econômica dessa implementação. A construtora contratou uma empresa de TI especializada nesse tipo de processo e tinha como objetivo gerir informações de produção com o máximo de eficácia. No final de 2013, implementou um novo sistema de gestão de informações, dirigido pela empresa especialista em gestão de processos de TI e negócios. A aplicação contou com acesso à internet baseado nas necessidades da obra, tendo como uma das principais ferramentas os tablets. De imediato, foi possível notar algumas melhorias, principalmente na redução de retrabalhos causado por imprecisão de informação. Por meio da solução, foi possível analisar o uso de materiais para evitar desperdícios, como no uso do gesso, do piso e da cerâmica nos apartamentos. Foi também estabelecido um cronograma mais eficaz, e evitou-se que erros fossem percebidos somente no momento de entrega das chaves ao cliente. (MOBUSS CONSTRUÇÃO, 2014)

De acordo com Nascimento e Santos (2003), os empecilhos principais a implementação de mudanças tecnológicas no canteiro de obra são a dificuldade de transformação do modelo de gestão atual e os problemas relacionados à introdução dessas tecnologias no canteiro de obra. O argumento dos autores supracitados é coerente, pois mesmo diante da popularização dos dispositivos móveis, atrelada à redução do custo de aquisição desses equipamentos, essa transformação ainda é pouco visível. Os exemplos de outros setores da economia nacional que têm obtido êxito no uso de dispositivos móveis visando a melhoria do fluxo da informação nos seus setores conferem maior segurança aos investimentos da construção civil nesse sentido. Mesmo assim, é perceptível que a introdução de dispositivos móveis no canteiro de obras brasileiro em substituição das pranchas de desenho implica em mudanças nos processos de projeto e no perfil do fluxo da informação no canteiro de obra, tornando necessárias mudanças na gestão. Além do mais, a utilização de modelos BIM para dispositivos móveis só faz sentido se pensado dentro de um plano estratégico de implementação BIM, o que significa mudanças maiores ainda de gestão, de processos e de tecnologias.

Este trabalho buscou contribuir de forma prática na já conhecida questão de adequação do BIM à realidade brasileira por meio da análise de opções de visualização em meio digital, uma questão significativamente importante para uma implementação eficaz e eficiente. $O$ estudo sugestivo e comparativo gerado ainda será usado nas fases seguintes da pesquisa como base para os testes em canteiros de obras, previstos como desenvolvimento desse tema.

\section{Conclusões}

Concluiu-se que a permanência da informação no meio digital, mesmo durante a fase executiva da obra propicia um melhor aproveitamento das informações do modelo BIM, permitindo a extração completa e precisa da informação do edifício, sem a necessidade de uso de desenhos abstratos, imprecisos e com "pouca inteligência" (as alterações no projeto não são atualizadas no papel impresso), além de evitar os gastos corriqueiros de impressão de desenhos.

O emprego de dispositivos móveis tem como grande vantagem em relação às plantas impressas a mobilidade de locomoção no canteiro e o baixo custo de investimento (a longo prazo). Além disso, a mobilidade de dados possibilitada por essas soluções, que são capazes de sincronizar e atualizar informações de projeto através da internet, (AMARAL; DUARTE, 2012) é capaz de melhorar significativamente o fluxo de informações entre o escritório de projeto e canteiro, além de melhorar a qualidade da informação disponível no canteiro de obras. O conhecimento das opções de aplicativos de visualização disponíveis é parte importante na tomada de decisões relacionadas ao uso (mesmo que parcial) de informação digital no canteiro de obras e é nesse contexto que este trabalho espera ter contribuído.

\section{Referências}

Amaral, M. C., Duarte, G. D. (2012). Desenvolvimento de um Aplicativo Web para Gerenciamento de Obras de Construção Civil para Uso em Tablets. Congresso de Iniciação Científica UFPel. Andrade, M. L., Ruschel, R. C. (2009). BIM: conceitos, cenário das pesquisas publicadas no Brasil e tendências. Simpósio Brasileiro de Qualidade do Projeto no Ambiente Construído.

Delatorre, J. P. M., Santos, E. T. (2014). Introdução de Novas Tecnologias: O Caso do BIM em Empresas de Construção Civil. XV Encontro Nacional de Tecnologia Do Ambiente Construído. DoNascimento, L. A., Santos, E. T.(2003). A indústria da construção na era da informação. In Ambiente Construído (v. 3, n. 1, pp. 69-81) Eastman, C. et al. (2011). BIM Handbook: A guide to building information modeling for owners, managers, designers, engineers and contractors.

FONTES, Gustavo Pacheco; SOARES, Paula Meyer. (2015). O Uso dos Tablets nas Organizações: Analise do Impacto no Ambiente de Trabalho. Revista Metropolitana de Sustentabilidade (v. 2, n. 1, p. 3-19).

Goes, R., Santos, E. T. (2011). Compatibilização de Projetos: Comparação entre o BIM e CAD 2D. V Encontro de Tecnologia de Informação e Comunicação na Construção.

Mobuss Construção. (2014). Construtora Frechal utiliza Mobuss Construção e reduz retrabalho em até 70\%. Disponível em http:// www.mobussconstrucao.com.br/2014/11/14/case-de-sucessoconstrutora-frechal

Neto, R. N., Ruschel, R. C., Picchi, F. A. (2013). Avaliação de ferramentas de tecnologia da informação na construção com funcionalidades móveis compatíveis aos itens da NBR ISO 9001: 2008. Revista Eletrônica de Engenharia Civil (v. 6, n. 1, 2013) Scheer, S. et al. (2007). Impactos do uso do sistema CAD geométrico edouso do sistemaCAD-BIMnoprocesso de projetoem escritórios de arquitetura. VII Workshop Brasileiro de Gestão do Processo de Projetos na Construção de Edifícios (Curitiba, UFPR) 